\title{
Into the Storm: a Mixed Methods Evaluation of Reasons for Non-attendance of Appointments in the Free Clinic Setting
}

\author{
Lydia K. Bedford $^{1} \cdot$ Collin Weintraub $^{1} \cdot$ Alan W. Dow ${ }^{2}$ (D)
}

Accepted: 11 October 2020 / Published online: 15 October 2020

(C) Springer Nature Switzerland AG 2020

\begin{abstract}
Non-attendance of healthcare appointments impact individual health outcomes and the capacity and financial stability of clinics. While non-attendance of appointments has been associated with a variety of factors, interventions to increase attendance have had mixed success. The most widely used intervention, reminder systems like phone calls or text messages, generally improves attendance rates but is insufficient for many clinics as a sole intervention. This study of underresourced patients who did not attend appointments at two clinics for uninsured individuals describes the multifactorial, individualized, and interacting reasons for non-attendance among these methods: Forty-three patients were interviewed by phone within 3 weeks of missing a clinic appointment using a scripted interview based on the literature. Responses were coded and analyzed. For $57 \%$ of respondents, a competing priority such as work or caregiving was a reason for missing an appointment. Forgetting about the appointment was a barrier for $38 \%$ of participants despite reminder systems being in place. Contributions to non-attendance were identified through thematic analysis: emotional and physical exhaustion, prioritization of work over healthcare, unreliable transportation, financial stress, and being unaware of an appointment. These findings demonstrate the need to test multiple patient-centered interventions, particularly in the context of underresourced communities.
\end{abstract}

Keywords Appointments and schedules · Health services accessibility $\cdot$ Medically uninsured $\cdot$ Vulnerable populations . Continuity of patient care $\cdot$ Primary care $\cdot$ Ambulatory care facilities $\cdot$ Reminder systems $\cdot$ Patient navigation

\section{Introduction}

Non-attendance of scheduled medical appointments is a concern for medical providers and policy-makers (Fig. 1). Nonattendance is an indicator of a failure of access to healthcare as described by the conceptual framework of Aday and

This article is part of the Topical Collection on Medicine

Alan W. Dow

alan.dow@vcuhealth.org

Lydia K. Bedford

bedfordlk@vcu.edu

Collin Weintraub

weintraubc@mymail.vcu.edu

1 School of Medicine, Virginia Commonwealth University, Richmond, VA, USA

2 School of Medicine, Virginia Commonwealth University, Box 980071, 1301 E. Marshall, VA 23298-00071 Richmond, USA
Andersen [1]. Non-attendance has been associated with worse control of patients' chronic disease, presumably through reductions in the continuity of care $[2,3]$. It also can negatively impact the clinic's population as a whole by reducing the availability of appointments to other patients who could have been scheduled during that time. Non-attendance also can have financial implications for clinics depending on the clinic's care delivery model and payment sources [4]. Because a myriad of clinic, patient, and environmental factors may influence access to healthcare and, potentially, nonattendance [1], understanding these factors and developing focused interventions is necessary to increase attendance rates.

Prior studies have associated many characteristics of clinics, appointments, and patients with non-attendance. Clinic and appointment factors identified include the type of provider [5], specific specialties [5-7], day of the week [5, 7], and a longer time between when the appointment is scheduled and the appointment date [5-7]. Patient factors that have been identified include behavioral or circumstantial characteristics, such as having a past history of non-attendance of 
Fig. 1 Conceptual framework of causes and results of nonattendance of appointments

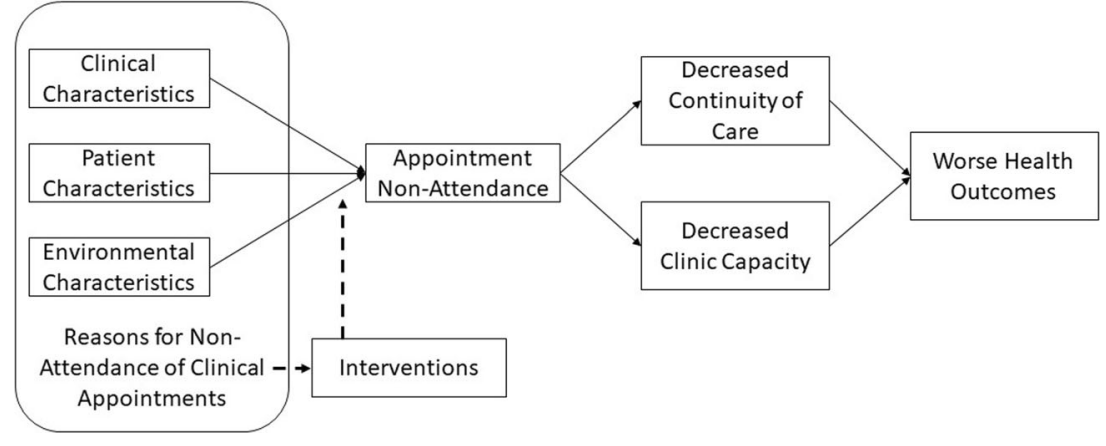

appointments [5-8], smoking tobacco [6-8], not owning a cellphone [7], and being publicly insured or paying out-of-pocket [6-8], as well as intrinsic characteristics such as age [5-8], race and ethnicity [6-8], and gender [5-7]. However, there is a wide discrepancy in findings across studies, and even across clinics within a single health system [7]. When patients have been asked about reasons for non-attendance at clinical appointments, studies have identified a variety of reasons including transportation problems [9-11], financial difficulties [11], being unable to take time from work $[9,12,13]$, childcare and family commitments [9, 14], long clinic wait times $[9,11]$, being hospitalized or too ill to attend [9-11], prioritizing other health problems [12], and forgetting $[10-12,14]$.

Better understanding the factors that contribute to non-attendance is critical for developing interventions to increase attendance rates. The most common intervention, reminder systems via phone calls and text messaging, has been widely studied internationally and is largely successful in increasing appointment attendance in a large variety of settings $[15,16]$. However, responses vary widely across sites [15], and reminder systems have shown to not solve the problem of non-attendance, likely because of the multiple factors that may lead to non-attendance. In addition, patients in underresourced settings may have greater and more complex challenges and require different solutions than those patient populations typically studied in this arena.

The aim of this research was to explore reasons for nonattendance by patients of two free clinics in Virginia, USA, through a scripted interview approach. The patients at these sites were mostly uninsured, in contrast to the existing literature where the majority of patients were insured $[9,14]$ or lived in countries with universal healthcare coverage [11]. Both clinics had implemented telephone reminders prior to the study period, but clinic staff noted that attendance rates continued to lag. This study sought to define the patientidentified reasons for non-attendance at these locations to guide future interventions that are patient centered and effective for underresourced populations.

\section{Methods}

\section{Study Setting and Population}

Individuals who did not attend appointments at the two free clinics ( $\mathrm{A}$ and $\mathrm{B}$ ) were contacted by phone to participate in the study over a 7-week period during the summer of 2019 . Inclusion criteria were missing an appointment during the study period, fluency in spoken English, 18 years of age or older, and having a phone number listed with the clinic. Clinic A provides both social and health services to its surrounding community. Clinic A serves uninsured and underinsured patients with primary care and specialty services in cardiology, rheumatology, psychiatry, and gynecology. Clinic B primarily serves patients with cardiovascular disease and its antecedents such as hypertension, hyperlipidemia, and diabetes, through a pharmacist-led model while also providing behavioral health services and other specialty services.

\section{Study Procedure}

Eligible participants were identified by staff or volunteers at each clinic when they indicated in the clinics' electronic health record (EHR) system that a patient was a "no show," a term used by clinics for non-attendance without a cancelation. All eligible participants were contacted by researchers by phone within 4 days of the appointment they did not attend. If reached by phone, eligible participants were first given an opportunity to reschedule their appointment. Next, they were asked to participate in the research study. For those individuals who agreed to participate, researchers attained informed consent and conducted a scripted interview (Appendix). The script began with the open-ended question, "Could you tell me about why you missed your appointment?" So that participants could speak in their own words. The interview proceeded with a series of "yes/no" questions designed to identify additional factors that may have contributed to nonattendance, based on reasons for non-attendance previously identified in the literature $[10,12,14,15]$. Participants were encouraged to expand upon their "yes" or "no" answers and these explanations were also recorded. The researchers asked 
clarifying questions as needed. Field notes were used in lieu of recordings and verbatim wordage to increase participants' trust of anonymize responses. All data was collected in a secured REDCap database. Eligible participants who did not answer initial calls were called again up to a total of three times over the next 3 weeks.

\section{Data Analysis}

After reviewing data for all participants, responses were grouped into categories of the primary reason for missing the appointment by study personnel ( $\mathrm{LB}$ and $\mathrm{CW}$ ). The groupings for this quantitative portion of the study were determined by the initial categories as listed in the interview script, with the exception of responses that did not fit any of the categories. The novel categories developed following data collection were "unable to contact clinic" and "didn't know about appointment." These categories were formed by two study personnel independently reviewing the data for themes and synthesizing their findings. Data was further reviewed for thematic analysis to develop themes from the qualitative data [17]. This study was approved by the Institutional Review Board at Virginia Commonwealth University.

\section{Results}

\section{Recruitment and Enrollment}

The outcomes of participant enrollment are depicted in Fig. 2. The sixty-nine eligible participants did not attend appointments over the 7-week period and all were contacted: 21 from clinic A and 48 from clinic B. Twenty-six of the eligible participants were never able to reach: six (9\%) of eligible participants could not be contacted due to a wrong phone number $(n=1)$, calling restrictions $(n=1)$, the call ending before ringing $(n=2)$, or number not in service $(n=2)$. Twenty (29\%) potential participants had an operational phone number but were never reached directly. In total, 43 individuals (62\% of eligible participants) were spoken to directly. Of these, 21 (30\% of the total population) were elected to participate in the study. Four additional individuals (9\% of the population) reported interest in the study but were unable to speak at the time of the call and were not reachable during later calls.

The time from the appointment to participation in the project ranged from 1 to 19 days, with an average of 7 days and a median of 6 days. Only one participant was interviewed for more than 2 weeks after their appointment.

\section{Primary Reasons for Non-attendance}

The primary reasons for non-attendance are shown in Fig. 3. The most common reason for non-attendance was having

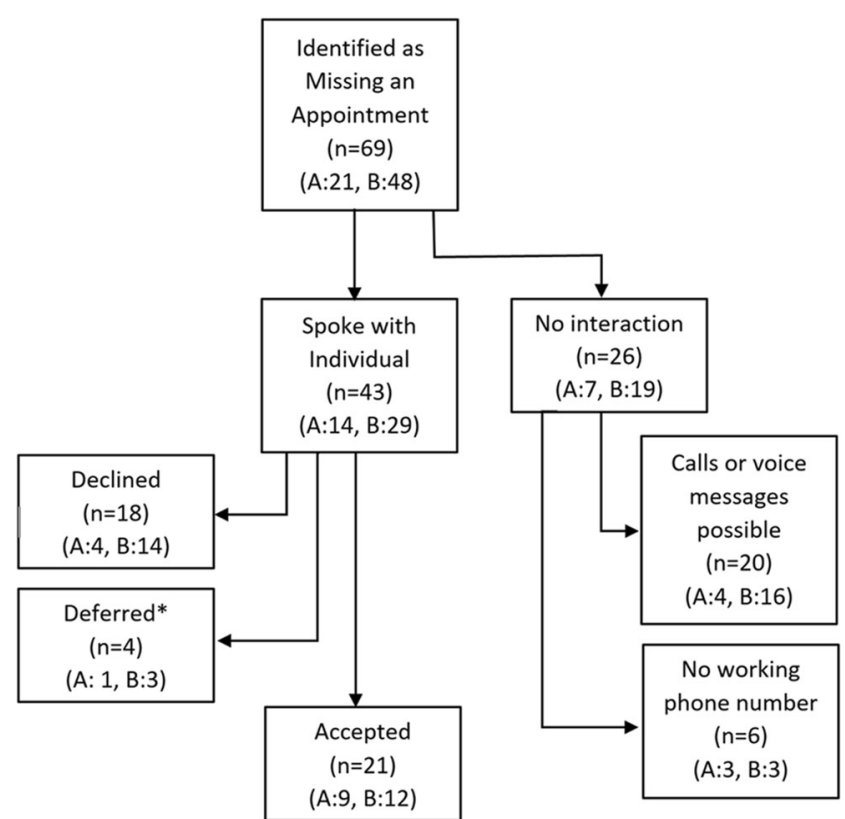

Fig. 2 Participant recruitment and enrollment. *Some patients expressed interest in participating in the research study but requested to speak at a later time. Of these, four were not reachable upon further calls. A, clinic A; B, clinic B

another priority ( $n=12,57 \%$ of respondents). Priorities that took precedence over clinic appointments were identified as working $(n=5)$, attending to family members $(n=4)$, both working and attending to family $(n=1)$, seeking assistance through social services $(n=1)$, and shopping $(n=1)$. Forgetting impacted eight participants in total $(n=38 \%)$, six of whom (29\%) reported that they also had another priority while two did not identify a competing priority (9\%). Other primary reasons for non-attendance that were identified by multiple people were a lack of transportation $(n=3,14 \%)$, going to a different provider $(n=1,5 \%)$, being unaware of having an appointment $(n=1,5 \%)$, and being unable to contact the clinic because they did not have the clinic phone number due to the appointment being scheduled by a local hospital or a probation officer $(n=2,9 \%)$. No participants reported missing their appointment for the following primary reasons: being unsure of the reason for their appointment, believing they did not need their appointment, feeling better, being unable to adhere to their doctors' orders, enrolling in insurance, or being unhappy with previous visits.

\section{Non-attendance Following Rescheduling}

Forty-eight (70\%) of the participants who were contacted scheduled an appointment subsequent to the appointment they did not attend. Of these patients, 21 (44\%) attended their next appointment, 19 (40\%) did not attend the next appointment, and $8(17 \%)$ canceled. 
Fig. 3 Primary reasons for nonattendance

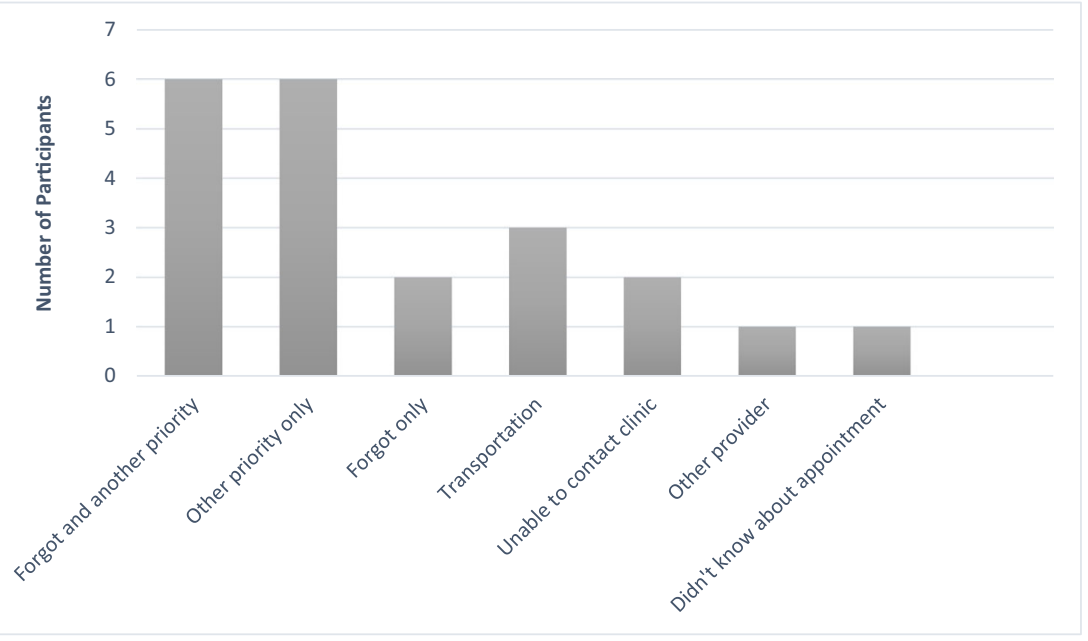

\section{Thematic Analysis}

Through the thematic analysis, the common themes identified were physical and emotional exhaustion, work needed to be prioritized over the appointment, travel barriers, and financial instability.

\section{Emotional and Physical Exhaustion}

Participants focused on the needs of family members during traumatic experiences. These included emotional caretaking for a family member recently diagnosed with cancer and watching over a family member who was in the hospital for weeks due to injuries from being shot. Participants also reported that their work took a toll on them even if they were not scheduled to work on the day of their appointment. Participants reported working 12-h shifts, working late or overnight, and working on their feet throughout their shift. Financial insecurity was overwhelming for one participant, who remarked that they constantly felt depressed and aggravated due to being unable to afford basic necessities.

\section{Prioritization of Work Over Healthcare}

Participants felt unable to take off from work to attend their appointment regardless of whether they were allowed to miss work. Taking time off to care for family the week before led one participant to miss their appointment as they felt that repeatedly taking time off would appear as a bad habit to their managers. Another started a new job the week of their appointment and was worried they would lose their job if they took time off on their third day of work. When one participant was running late in the morning, they chose to prepare for work over attending their appointment as they did not feel that the clinic appointment would be an acceptable excuse for missing time from work.

\section{Unreliable Transportation}

Lack of adequate transportation was a barrier for patients whether they lived near to or far from the clinics. Some have experienced having no driver's license or no car, having their car break down, or sharing one care between three people which limited all three people's ability to travel easily. Friends' and relatives' availability to transport them to clinic appointments was often unreliable. Having no gas money further prevented travel even with a car available. Bus routes were not convenient for some people who relied on public transportation for travel, and some did not reliably have money for bus fare.

\section{Financial Stress}

Lack of financial means extended beyond transportation and led to them diverting their time and money from clinic appointments out of necessity. A participant spent much of their money while unemployed on the weekly cost of a hotel room where they were currently living. Another needed all of their money to pay for their bills because they were relying on receiving a payment which was postponed. Being unable to contact social services for public insurance and other benefits by phone necessitated a participant to spend the day of their appointment at the social services office. They decided that securing insurance would benefit them most although it necessitated missing an appointment for treatment of their chronic health condition.

\section{Being Unaware of an Appointment}

While some participants forgot their appointment entirely, others were only aware that they had an appointment once they received a reminder call. Others believed their appointment was scheduled for one date or time that was inconsistent 
with the clinics' records. Reminder calls were too late for the participants who did not attend their appointment leaving them unable to attend or reschedule.

\section{Discussion}

This study sought to determine reasons for non-attendance among patients at two free clinics that employed reminder phone call systems prior to the start of the study. The aim was to identify areas for improvement in appointment attendance, both to increase patients' access to care and improve the clinic's efficiency. This study demonstrated that reasons for non-attendance among this population are varied and often multifactorial. The predominant primary reasons for nonattendance were having another obligation at the time of the appointment and forgetting despite a reminder phone call system already in place. Notably, during participant recruitment, $29 \%$ of eligible participants had a working phone number but did not respond to calls or voicemails. This might indicate general disinterest in communication with the clinic or that phone calls are not a preferred or reliable method of contact for some patients. Ideally, reminder systems are tailored to patients which might include text messaging, e-mails, and patient portal messages, and multiple messages for patients to have more time to cancel or reschedule if needed $[15,16]$. Based on this study, where additional obligations such as the need to work or care for loved ones impacted the majority of participants, additional approaches that are patient-centered are necessary to improve appointment attendance. These results should also help to familiarize clinic staff and volunteers, particularly those who are new to working with underresourced individuals, with the extensive barriers to attendance that these patients face.

Transportation was a common theme and the primary reason for non-attendance in $14 \%$ of participants. Travel barriers were typically intermittent and associated with insufficient funds to travel, and financial assistance such as vouchers for bus fare or gasoline might have helped some of the participants who faced transportation barriers. One-day bus tickets cost a maximum of $\$ 3.50$ within the cities where the clinics are located, indicating that cost to the clinics to help patients overcome financial transportation barriers may be feasible. This would likely not help all patients, however, given that those who live a considerable distance from the clinics might require very expensive assistance which in a previous study was found not to increase the attendance rate [18]. Patientcentered education about local transportation could be an additional approach without cost to the clinics. One year before this study, the bus system in the city where clinic B is located reorganized bus routes, including closing the bus stop that was closest to the clinic, which one participant expressed difficulty navigating. Flyers with information on bus routes and training of front desk staff and volunteers to guide patients through the new bus system by phone might increase the accessibility of the clinic to patients. This appears to be a novel area for future research.

Patient navigators are a resource-intensive approach to increase attendance rates but have been successful at some clinics $[8,11,19]$, with navigators being able to both identify reasons for non-attendance and improving patients' understanding of their need for appointments through personalized education. A major challenge to implementing this approach is the financial cost of a navigator to the clinic because they must be well educated on the services available at the clinic and in the community, as well as having a considerable time to work with patients to access these services. A more costeffective approach could be a limited patient navigator role in which the navigator spends time only working with patients who regularly miss appointments, such as the $57 \%$ of the participants in our sample who asked to be rescheduled but did not attend their subsequent appointment.

Finally, telemedicine may be a promising approach to address issues related to travel and competing demands from work, family, and other sources, and particularly with the COVID-19 pandemic, though this study was conducted for the start of the pandemic. Telemedicine has increased appointment attendance among underresourced patients [20,21]. This area warrants further study.

\section{Limitations}

Limitations in this study include that the majority of eligible participants did not participate in the study due to either declining participation or being unreachable by phone. This is a common finding in similar studies $[8,10,12]$, and this population likely represents an essential group to study further. In addition, though the interview guides "yes or no" questions were based on the literature, they might have limited participants' responses to the researchers' areas of focus. It is possible that there are institutional and societal factors influencing appointment adherence that were neither reported by participants in the open-ended question nor asked directly by the researchers. Response bias due to participants' concerns over anonymity might also affect results, though participants were informed that their responses would be de-identified and clinic staff and volunteers would have no access to identifying information. Recall bias due to delay in communication with participants between the non-attended appointment and the interview might also have an impact.

\section{Conclusion}

Non-attendance of appointments is common and detrimental to patients and clinics $[2-4,6]$. This study contributes to the understanding of patients' reasons for 
non-attendance in the context of underresourced settings which already have a reminder system in place. A wide range of causes and the multifactorial nature of nonattendance present considerable challenges to clinics seeking to increase appointment attendance. Interventions including reminder systems that are better tailored to patient preferences, transportation vouchers, patient navigator programs, and telemedicine have both promise and shortcomings and are important areas for further study.

\section{Compliance with Ethical Standards}

This study was approved by the Institutional Review Board at Virginia Commonwealth University.

\section{Appendix: Interview script}

1) First, could you tell me about why you missed your appointment?

2) Did you feel you did not need your appointment anymore?

3) Were you feeling better and felt that you did not need to see the doctor?

4) Often people aren't able do everything the doctor orders, like pick up medications, get lab tests, or make changes at home. Did any of those happen to you?

5) Did you go somewhere else instead?

6) Did you get insurance?

7) Were you unsure what the appointment was for?

8) Did you want to cancel or reschedule the appointment, but you were not able to get through to the clinic on the phone?

9) Were you unhappy with previous visits?

10) Did you have anything you needed to do that day that kept you from coming in?

(If yes):

a. Did you have kids or anyone else you had to look after?

b. Were you unable to take time off from work or school?

11) Did you have trouble getting to the clinic?

12) Were you unable to get a ride or pay for bus fare?

13) Did you have trouble affording the clinic? (If yes):

a. Were you unable to pay to get to the clinic, like paying bus fare?

b. Were you unable to pay for donations or medications at the clinic?

c. Was there anything else you could not pay for that kept you from coming in?
14) Were you feeling too sick or unwell to come in?

15) Did you forget about the time or day of the appointment?

16) Were there any other reasons you did not make it to the appointment?

\section{References}

1. Aday LA, Andersen R. A framework for the study of access to medical care. Health Serv Res. 1974;9(3):208-20.

2. Karter AJ, Parker MM, Moffet HH, Ahmed AT, Ferrara A, Liu JY, et al. Missed appointments and poor glycemic control: an opportunity to identify high-risk diabetic patients. Med Care. 2004;42:110 5. https://doi.org/10.1097/01.mlr.0000109023.64650.73.

3. Schectman JM, Schorling JB, Voss JD. Appointment adherence and disparities in outcomes among patients with diabetes. J Gen Intern Med. 2008;23(10):1685-7. https://doi.org/10.1007/s11606008-0747-1.

4. Bech $\mathrm{M}$. The economics of non-attendance and the expected effect of charging a fine on non-attendees. Health Policy (Amsterdam, Netherlands). 2005;74(2):181-91. https://doi.org/10.1016/j. healthpol.2005.01.001.

5. Torres O, Rothberg MB, Garb J, Ogunneye O, Onyema J, Higgins T. Risk factor model to predict a missed clinic appointment in an urban, academic, and underserved setting. Popul Health Manag. 2015;18(2):131-6. https://doi.org/10.1089/pop.2014.0047.

6. Dantas LF, Fleck JL, Cyrino Oliveira FL, Hamacher S. No-shows in appointment scheduling - a systematic literature review. Health Policy. 2018;122(4):412-21. https://doi.org/10.1016/j.healthpol. 2018.02.002.

7. Mohammadi I, Wu H, Turkcan A, Toscos T, Doebbeling BN. Data analytics and modeling for appointment no-show in community health centers. J Prim Care Community Health. 2018;9: 2150132718811692. https://doi.org/10.1177/2150132718811692.

8. Luckett R, Pena N, Vitonis A, Bernstein MR, Feldman S. Effect of patient navigator program on no-show rates at an academic referral colposcopy clinic. J Women's Health (Larchmt). 2015;24(7):608 15. https://doi.org/10.1089/jwh.2014.5111.

9. Tofighi B, Grazioli F, Bereket S, Grossman E, Aphinyanaphongs Y, Lee JD. Text message reminders for improving patient appointment adherence in an office-based buprenorphine program: a feasibility study. Am J Addict. 2017;26(6):581-6. https://doi.org/10. 1111/ajad.12557.

10. Shahab I, Meili R. Examining non-attendance of doctor's appointments at a community clinic in Saskatoon. Can Fam Physician. 2019;65(6):e264-8.

11. Brewster S, Bartholomew J, Holt R, Price H. Non-attendance at diabetes outpatient appointments: a systematic review. Diabet Med. 2020;37(9):1427-42. https://doi.org/10.1111/dme.14241.

12. Bhise V, Modi V, Kalavar A, Espadas D, Hanser L, Gould M, et al. Patient-reported attributions for missed colonoscopy appointments in two large healthcare systems. Dig Dis Sci. 2016;61(7):1853-61. https://doi.org/10.1007/s10620-016-4096-3.

13. Martin C, Perfect T, Mantle G. Non-attendance in primary care: the views of patients and practices on its causes, impact and solutions. Fam Pract. 2005;22(6):638-43. https://doi.org/10.1093/fampra/ cmi076.

14. Kumthekar A, Johnson B. Improvement of appointment compliance in an underserved lupus clinic. BMC Health Serv Res. 2018;18(1):610. https://doi.org/10.1186/s12913-018-3429-7.

15. McLean S, Booth A, Gee M, et al. Appointment reminder systems are effective but not optimal: results of a systematic review and 
evidence synthesis employing realist principles. Patient Prefer Adherence. 2016;10:479-99.

16. Robotham D, Satkunanathan S, Reynolds J, Stahl D, Wykes T. Using digital notifications to improve attendance in clinic: systematic review and meta-analysis. BMJ Open. 2016;6(10):e012116. https://doi.org/10.1136/bmjopen-2016-012116.

17. Braun V, Clarke V. Using thematic analysis in psychology. Qual Res Psychol. 2006;3:77-101. https://doi.org/10.1191/ 1478088706qp063oa.

18. Chaiyachati KH, Hubbard RA, Yeager A, Mugo B, Lopez S, Asch $\mathrm{E}$, et al. Association of rideshare-based transportation services and missed primary care appointments: a clinical trial. JAMA Intern Med. 2018;178(3):383-9. https://doi.org/10.1001/jamainternmed. 2017.8336 .

19. Adams JA, Whiteman K, McGraw S. Reducing missed appointments for patients with HIV: an evidence-based approach. J Nurs
Care Qual. 2020;35(2):165-70. https://doi.org/10.1097/ncq. 0000000000000434.

20. Dayal P, Chang CH, Benko WS, Ulmer AM, Crossen SS, Pollock $\mathrm{BH}$, et al. Appointment completion in pediatric neurology telemedicine clinics serving underserved patients. Neurol Clin Pract. 2019;9(4):314-21. https://doi.org/10.1212/CPJ. 0000000000000649 .

21. Jani PD, Forbes L, Choudhury A, Preisser JS, Viera AJ, Garg S. Evaluation of diabetic retinal screening and factors for ophthalmology referral in a telemedicine network. JAMA Ophthalmol. 2017;135(7):706-14. https://doi.org/10.1001/jamaophthalmol. 2017.1150.

Publisher's Note Springer Nature remains neutral with regard to jurisdictional claims in published maps and institutional affiliations. 